\title{
RETRACTED ARTICLE: Application of cognitive system engineering in development of dragon boat sports
}

\author{
Zhi Zhang ${ }^{1} \cdot$ Li-Hong Hou ${ }^{1}$ \\ Published online: 17 June 2020 \\ (C) Springer Science+Business Media, LLC, part of Springer Nature 2020
}

The Editor-in-Chief has retracted this Article. The article was accepted as part of a guest-edited special issue in Current Psychology. Before the special issue was finalized, the Editor-in-Chief detected problems with editorial handling and peer review and decided not to proceed with the special issue.

Post publication peer review found the following issues:

- The main systems this article speaks to are not addressed. For example, although there has been much work done on cognitive systems engineering this article does not give any overview of how such integrated systems work.

- Other models this article undertakes to probe are not described at all (i.e. spatial planning model, game analysis, cognitive system control model).

- Methodologically, the article does not truly state how these problems are addressed. It is unclear methodologically how these models and equations for the models came to be.
- In the figures, the axis are labelled but not explained, nor are they explained fully in the text itself. These errors persist throughout the article.

- The methods are unclear as to answering the purpose of this study. It is unclear as to what the full data actually are, to conclude the purposes of this study.

The Editor-in-Chief therefore no longer has confidence in the reliability of the work presented.

Dr. Li-Hong Hou does not agree to this retraction. Dr. Zhi Zhang has not responded to any correspondence from the editor about this retraction.

The online version of this article contains the full text of the retracted article as Supplementary Information.

Supplementary Information The online version of this article (https:/doi. org/10.1007/s12144-020-00824-y) contains supplementary material, which is available to authorized users.
Li-Hong Hou

jlbh7777@126.com

1 College of Physical Education, Beihua University, Jilin, China 